\title{
Microbiota intestinal e câncer colorretal: uma revisão bibliográfica
}

\author{
Intestinal microbiota and colorectal cancer: a literature review \\ Microbiota intestinal y cáncer colorrectal: revisión de la literatura
}

Luiza Miranda Marinho de Paula ${ }^{1 *}$, Aléxia Sousa Guimarães², Ana Carolina Ramalho dos Reis ${ }^{3}$, Eliseu Gabriel Santos Lima ${ }^{2}$, Italo Matheus Dias de Andrade ${ }^{4}$, Matheus Naves Rosa ${ }^{5}$, Thaís Ruela Martins², Thamara Carolina Lobo Alves², Vitor Azevedo Sarmento², Isabella Lopes Lusvarghi6.

\section{RESUMO}

Objetivo: Analisar as evidências encontradas nas publicações científicas sobre a associação entre as comunidades bacterianas e o risco de Câncer Colorretal (CCR). Métodos: Trata-se de uma revisão sistemática de literatura utilizando ensaios clínicos randomizados ou não randomizados obtidos nas bases de dados PubMed e EMBASE que abordassem o tema sobre microbiota intestinal e câncer colorretal em adultos e idosos, publicados entre 2015 e 2020. Resultados: Identificou que Bifidobacterias spp e Lactobacillus spp atuam reduzindo o risco de câncer de cólon, Fusobactérias nucleatum poderia ser utilizada como um marcador para detecção de CCR e Eubacterium , Roseburia e Fusobacterium nucleatum possuem efeito carcinogênico na região colorretal. Considerações Finais: Foi analisado que um grupo de bactérias está consistentemente associado ao câncer colorretal, destacando-se biomarcadores promissores para a detecção precoce e não invasiva de neoplasia colorretal, tornando-se possível iniciar o tratamento em estágios iniciais, diminuindo a morbimortalidade. Além da identificação de colônias bacterianas capazes de reduzir o risco de câncer de cólon, melhorar o tratamento e a recuperação no pós-operatório.

Palavras-chave: Microbiota, Microflora, Câncer colorretal.

\begin{abstract}
Objective: To analyze the evidence found in scientific publications on the association between bacterial communities and the risk of Colorectal Cancer (CCR). Methods: This is a systematic review of the literature using randomized or nonrandomized clinical trials obtained from the PubMed and EMBASE databases that addressed the topic of intestinal microbiota and colorectal cancer in adults and the elderly, published between 2015 and 2020. Results: Identified whereas Bifidobacterias spp and Lactobacillus spp act to reduce the risk of colon cancer, Fusobacteria nucleatum could be used as a marker for the detection of CCR and Eubacterium, Roseburia and Fusobacterium nucleatum have a carcinogenic effect in the colorectal region. Final Considerations: It was analyzed that a group of bacteria is consistently associated with colorectal cancer, highlighting promising biomarkers for the early and non-invasive detection of colorectal neoplasia, making it possible to start treatment in early stages, decreasing morbidity and mortality. In addition to the identification of bacterial colonies capable of reducing the risk of colon cancer, improving treatment and recovery in the postoperative period.
\end{abstract}

Keywords: Microbiota, Microflora, Colorectal câncer.

\footnotetext{
1 Pontifícia Universidade Católica de Minas Gerais - Unidade Betim (PUC-MG), Betim - MG,

*E-mail: luizammarinho@hotmail.com

${ }^{2}$ Centro Universitário Governador Ozanam Coelho (UNIFAGOC), Ubá - MG.

${ }^{3}$ Centro Universitário de Patos de Minas (UNIPAM), Patos de Minas - MG.

${ }^{4}$ Universidade Federal do Rio de Janeiro (UFRJ), Rio de Janeiro - RJ.

${ }^{5}$ Universidade de Araraquara (UNIARA), Araraquara - SP.

${ }^{6}$ Faculdade de Minas (FAMINAS), Belo Horizonte - MG.
} 


\section{RESUMEN}

Objetivo: Analizar la evidencia encontrada en publicaciones científicas sobre la asociación entrecomunidades bacterianas y el riesgo de cáncer colorrectal (CCR). Métodos: Esta es una revisión sistemática de la literatura que utiliza ensayos clínicos aleatorios o no aleatorios obtenidos de las bases de datos PubMed y EMBASE que abordaron el tema de la microbiota intestinal y el cáncer colorrectal en adultos y ancianos, publicados entre 2015 y 2020. Resultados: Identificados Mientras que Bifidobacterias spp y Lactobacillus spp actúan para reducir el riesgo de cáncer de colon, Fusobacterium nucleatum podría usarse como un marcador para la detección de CCR y Eubacterium, Roseburia y Fusobacterium nucleatum tienen un efecto cancerígeno en la región colorrectal. Consideraciones Finales: Se analizó que un grupo de bacterias se asocia constantemente con el cáncer colorrectal, destacando los marcadores prometedores para la detección temprana y no invasiva de la neoplasia colorrectal, lo que permite comenzar el tratamiento en las primeras etapas, disminuyendo la morbilidad y la mortalidad. Además de la identificación de colonias bacterianas capaces de reducir el riesgo de cáncer de colon, mejorando el tratamiento y la recuperación en el postoperatorio.

Palabras clave: Microbiota, Microflora, Cáncer colorrectal.

\section{INTRODUÇÃO}

Segundo Campos FGCM, et al. (2017); o câncer colorretal (CCR) é o tumor maligno mais comum do trato gastrointestinal, sendo o segundo tipo de câncer mais frequente nas mulheres e o terceiro mais comum nos homens, com 1,8 milhão de casos novos e cerca de 862.000 óbitos no ano de 2018. No Brasil, isso se repete, sendo o segundo tumor de localização primária com maior incidência entre as neoplasias no sexo feminino $e$ terceiro lugar em incidência no sexo masculino. De acordo com as estatísticas desse mesmo instituto, foram observados 18.867 óbitos em 2017 e são esperados 38.230 novos casos em 2020 nesse país (CANCER, 2018; ESTATÍSTICA DO CÂNCER, 2020).

O câncer colorretal é uma patologia mundialmente comum e letal que atinge com maior prevalência grupos de idade mais avançada (CAMPOS FGCM, et al., 2017). Os principais fatores de risco relacionados com tal neoplasia são: sobrepeso, sedentarismo, idade igual ou superior a 50 anos, tabagismo, alimentação rica em gorduras e pobre em fibras, vegetais e frutas, ingestão de bebidas alcoólicas, grande consumo de carnes vermelhas e processadas, além dos fatores genéticos que estão envolvidos em 5-15\% dos casos (PELIZZER T, et al., 2016; GAO Z, et al., 2015).

As manifestações clínicas dessa neoplasia variam conforme a localização e o tipo de tumor. Quando localizado no cólon esquerdo, manifesta com alteração do hábito intestinal. Já nos tumores do cólon direito, aparecem sintomas como diarreia, anemia ferropriva e massa palpável no quadrante inferior direito. Nos tumores localizados no reto, é observado sangramento, geralmente vermelho vivo, e tenesmo que é a sensação de eliminação incompleta, apesar do reto estar vazio (SILVA MD e ERRANTE PR, 2016). Calculase que no aparelho digestivo exista cerca de $10^{14}$ micro-organismos, sendo que mais de 1000 espécies são diferentes. Tem verificado que essa microbiota interage de forma benéfica com o hospedeiro, fabricando enzimas, vitaminas e proteínas, ajudando na modulação do sistema imune, na absorção de nutrientes e na fermentação de biomoléculas para disponibilização de energia (WAGNER NRF, et al., 2018).

A etiologia dessa doença ainda é incerta, porém estudos têm relacionado a exposição aos fatores de risco ambientais à alteração da microbiota intestinal, podendo estar associado à fisiopatologia do câncer colorretal. Essa flora intestinal é composta por bactérias que protegem o organismo humano e por bactérias patológicas. Quando há um desequilíbrio entre elas, pode ocorrer uma inflamação da parede intestinal e uma possível evolução futura para neoplasia (GAO Z, et al., 2015).

Há pouco tempo, foi apontado que a existência de Enterococcus faecalis, Streptococcus bovis, Fusobacterium nucleatum, Bacteroides fragilis enterotoxigênicos (ETBF) e Porphyromonas spp e a escassez de Eubacterium spp, Roseburia spp, Lactobacillus spp e Bifidobacterium spp estavam envolvidos no aparecimento de adenomas e câncer colorretal (REZASOLTANI S, et al., 2018). Logo, pacientes com câncer colorretal suplementados com probióticos - microorganismos vivos usados na prática clínica devido ao seu potencial de causar efeitos benéficos à saúde- têm uma redução significativa de bactérias maléficas como as de Fusobacterium e Peptostreptococcus e melhora efetiva na variedade da microbiota intestinal (WAGNER NRF, et al., 2018; GAO Z, et al., 2015). 
Além da alteração na microbiota, estudos têm demonstrado outros benefícios em relação ao uso de probiótico em pacientes que estão em tratamento de CCR. Polakowski CB, et al. (2018) identificaram que pacientes portadores de CCR que usaram pré e probióticos em associação, apresentaram estimulação da função intestinal, redução de complicações, do tempo de internação hospitalar e do uso de antibiótico, além de diminuição do estado inflamatório. Semelhante a isso, o estudo de Zaharuddin L, et al. (2019); mostraram que os pacientes que fizeram uso de probióticos após a cirurgia, exibiram redução significativa no nível de algumas citocinas pró-inflamatórias.

O estudo revisa e esclarece os conceitos sobre os efeitos negativos que podem ocasionar quando uma microbiota intestinal se encontra em desequilíbrio. Além disso, busca analisar e sumarizar as evidências encontradas nas publicações científicas sobre a associação entre microbiota e o risco de câncer colorretal, com o intuito de que esses dados possam acrescentar às pesquisas científicas e a tomada de decisões clínicas.

\section{MÉTODOS}

O presente estudo trata-se de uma revisão sistemática, realizado com base no PICO (Quadro 1) e consistiu na avaliação das referências eletrônicas obtidas nas bases de dados PubMed (National Library of Medicine and National Institutes of Health) e EMBASE no período de 15 a 22 de abril de 2020, como pode ser visto na Figura 1.

A busca feita nas bases de dados informadas foi realizada através do descritor "microbiota AND colorectal cancer" para o PUBMED e "microflora AND colorectal cancer" em title, abstract, autor keywords para o EMBASE. Os filtros utilizados na PubMed foram "Controlled Clinical Trial"; "Clinical Trial, Phase IV"; "Clinical Trial, Phase III"; "Clinical Trial, Phase II"; "Clinical Trial", "published in the last 5 years" e "humans", resultando inicialmente em um total de 25 artigos. Na EMBASE, os limites foram preenchidos com "controlled clinical trial]/lim OR [randomized controlled trial]" de 2015 a 2020, totalizando em 4 artigos.

Dos 25 artigos obtidos da PubMed, 18 foram excluídos por não cumprirem os critérios de inclusão que serão descritos a seguir. Na EMBASE, dos 4 artigos encontrados, 1 deles era duplicata do PubMed e dessa forma, foi excluído. Ao todo 10 artigos foram usados, 3 Ensaios Clínicos (EC) e 7 Ensaios clínicos randomizados $(E C R)$.

Os critérios de inclusão definidos previamente foram: artigos de ensaio clínico randomizados ou não randomizados, que abordassem o tema sobre microbiota intestinal e câncer colorretal em adultos e idosos, publicados nos últimos 5 anos. Os critérios de exclusão: os artigos que tinham desenho metodológico diferente de ensaio clínico, os que não abordavam sobre o tema, os que tratavam de transplante de fezes e não contemplavam a idade dos participantes.

Quadro 1 - Pergunta PICO - Caracterização dos pacientes atendidos em unidades secundárias de atenção à saúde, sem limitação quanto à amostra e a localização.

\begin{tabular}{|c|c|}
\hline População & $\begin{array}{c}\text { Pacientes com câncer colorretal atendidos em unidades secundárias de } \\
\text { atenção à saúde, sem limitação quanto à amostra e a localização. }\end{array}$ \\
\hline Intervenção/interesse & $\begin{array}{c}\text { Avaliar se a presença de comunidades bacterianas influencia o câncer } \\
\text { colorretal }\end{array}$ \\
\hline Comparação & Pacientes sem câncer \\
\hline O Desfecho & Associação ou não entre bactérias e o câncer colorretal \\
\hline
\end{tabular}

Fonte: De Paula LMM, et al., 2020. 
Figura 1 - Seleção de artigos

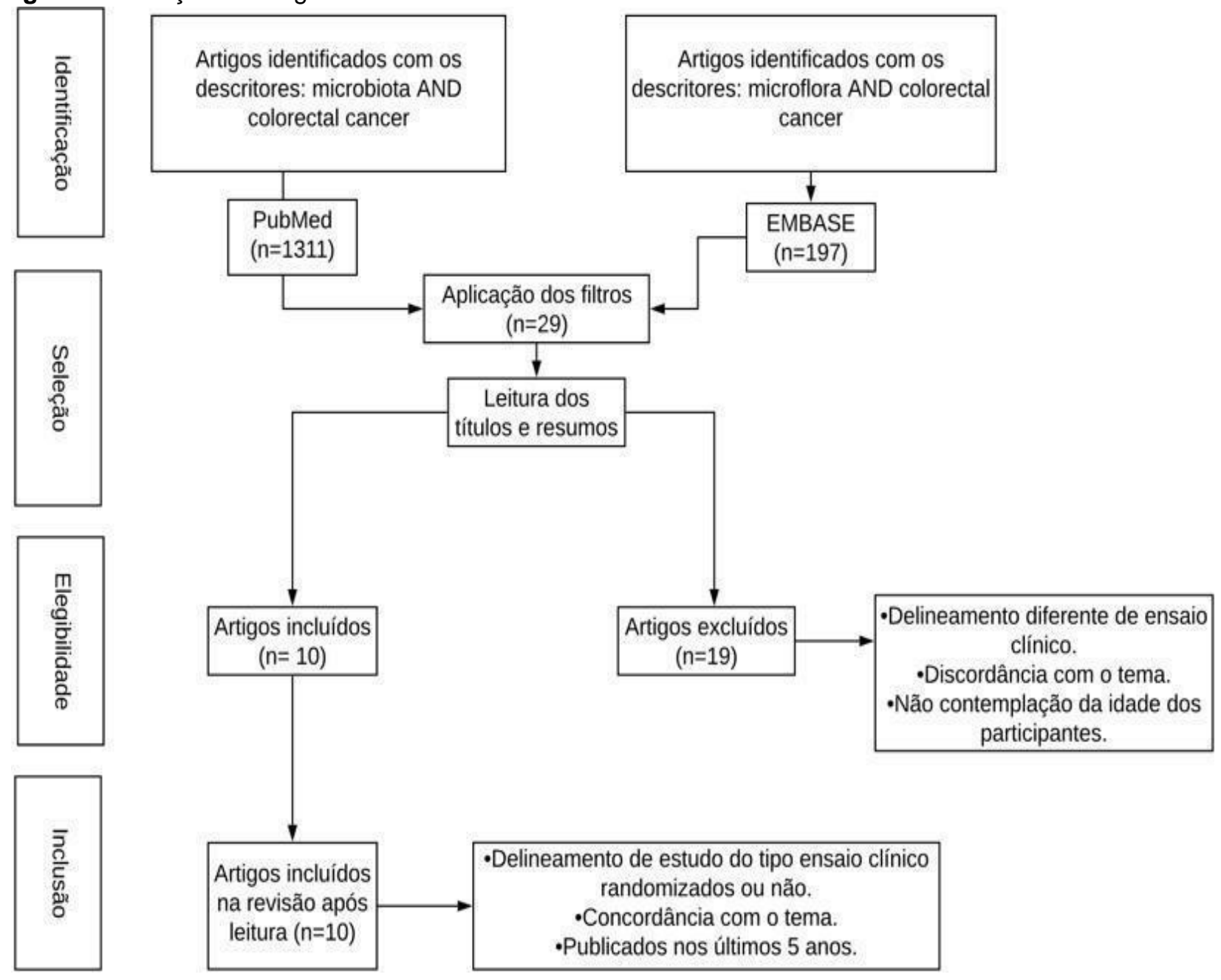

Fonte: De Paula LMM, et al., 2020.

\section{RESULTADOS}

Tendo vista os dados expostos no Quadro 2, Xiaoliang X, et al. (2019) avaliaram o efeito da suplementação de prebióticos no peri-operatório de 140 pacientes sabidamente portadores de câncer colorretal (CCR). Realizaram suplementação com $30 \mathrm{~g} /$ dia de prebióticos no pré operatório a qual aumentou os níveis séricos de Imunoglobulina $G(\lg G ; P=0,02)$, $\lg M(P=0,00)$ e transferrina $(P=0,027)$. No pósoperatório, foram identificados níveis aumentados de $\lg G(P=0,003), \lg A(P=0,007)$, células $T$ supresoras / citotóxicas $(C D 3+C D 8+; P=0,043)$ e linfócitos $B$ totais $(C D 19+; P=0,012)$.

Em relação à microbiota, no pré-operatório, também apresentaram aumento na abundância de alguns gêneros bacterianos, tais como Bifidobacterium $(P=0,017)$ e Enterococcus $(P=0,02)$, mas diminuíram a abundância de Bacteroides $(P=0,04)$. No pós-operatório, houve uma abundância de Escherichia-Shigella após a ingestão de prebióticos $(P=0,014)$. Em última análise, concluíram que a não suplementação prebiótica foi responsável pelo declínio da microbiota no período peri-operatório (XIAOLIANG X, et al., 2019).

A maioria dos pacientes com câncer colorretal apresentava níveis elevados de fatores inflamatórios e transferrina, que teve os níveis elevados após a suplementação. Além de ter diminuído a inflamação, o câncer e certas doenças. Zaharuddin L, et al. (2019) comprovam através do estudo, esse mesmo papel da modulação de fatores inflamatórios citado anteriormente. Nesse caso essa modulação foi constatada através do uso de probióticos, os quais melhoram o status imunológico e reduziram o tempo de internação hospitalar, infecção no local de cirurgia incisional, complicações pós cirúrgicas e diarreia induzida por antibióticos (XIAOLIANG X, et al., 2019). 
Sendo apontado como um complemento promissor para prevenção e tratamento do câncer colorretal. Foi observado que os pacientes que receberam os probióticos em comparação ao placebo $(P<0,05)$, tiveram redução significativa nos níveis de fator de necrose tumoral alfa (TNF- $\alpha)(P=0.002)$, interleucinas (IL)- 6 , IL 10 $(P=0.028), I L-12(P=0.005)$, IL-17 (ou IL-17A) $(P=0.000)$, IL-17C e IL $22(P=0.018)$ fornecendo resistência à morte celular e estimulando produção de citocinas pró-inflamatórias através das vias de sinalização, com isso, protegendo a camada gastrointestinal (XIAOLIANG X, et al., 2019).

Polakowski CB, et al. (2018) também descreveu o efeito da administração simbiótica pré-operatória em pacientes com CCR submetidos à ressecção colorretal. 8 dias antes da cirurgia, os pacientes foram randomizados para receber antibióticos (Simbioflora) ou placebo (maltodextrina). Após 7 dias da ingestão de antibióticos, houve reduções significativas nos níveis de IL-6 e proteína $C$ reativa, enquanto o grupo controle não apresentou alterações significativas. Concluíram que o uso de antibióticos por 7 dias no pré-operatório em pacientes com CCR atenua o estado inflamatório e está associado a reduções de morbidade, tempo de internação hospitalar e uso de antibióticos. (POLAKOWSKI CB, et al., 2018).

Outro estudo que concorda com o atenuamento inflamatório causado nesse caso por suplementação de $4 \mathrm{~g}$ de ácido eicosapentaenoico misto / ácido docosahexaenoico, é o Watson $\mathrm{H}$, et al. (2017). Essa suplementação foi ofertada em formulações de cápsulas e bebidas, as quais fornecem incorporação equivalente de ômega-3 teciduais. O aumento na densidade de Bifidobacterium, Lactobacillus e Roseburia conhecidas por serem produtoras de butirato, foi compatível com propriedades anti inflamatórias e anti neoplásicas (POLAKOWSKI CB, et al., 2018).

Em seu estudo piloto Borresen EC, et al. (2016) demonstrou que o aumento da ingesta de feijões da marinha (FM) ou farelo de arroz (FA), por sobreviventes de câncer colorretal, a níveis que anteriormente mostravam bio atividade quimiopreventiva é bastante viável, o que contribui para o aumento do consumo de fibra alimentar, niacina, tiamina, ferro, zinco, folato, vitamina B6 e alfa-tocoferol (BORRESEN EC. et al., (2016).

A fibra alimentar é um macronutriente de grande relevância que mostra evidências de redução do risco do câncer. Possivelmente os mecanismos dessa prevenção possuem inúmeras facetas, pois além da fibra contribuir para a saúde do intestino por reduzir o tempo do trânsito oro-fecal, ela produz ácidos graxos de cadeias curtas que por sua vez cooperam para a redução da inflamação intestinal (WATSON H, et al., 2017).

Pearson T, et al. (2018); utilizaram ácido ursodeoxiólico (UDCA), um ácido biliar sintético usado na clínica. Mudanças na microbiota composicional não foram apresentadas no grupo placebo, tendo resultado somente associados a comunidade no ácido ursodeoxiólico (UDCA). Foi observado que os dois microorganismos dominantes da microbiota intestinal foram Bacteroidetes e Firmicutes. Sendo assim, o UDCA pode reduzir o risco de adenoma colorretal, mas ainda precisa de mais estudos para determinar o mecanismo associado a microbiota.

Gao Z, et al. (2015) mostra que os patógenos, em potencial, como Fusobacterium e Peptostreptococcus, são significativamente enriquecidos na microflora da mucosa do câncer colorretal (CCR). Neste estudo, foi realizada a pirosequenciação para determinar como os probióticos alteram a microbiota e este demonstrou que os pacientes com CCR tratados com a administração oral obtiveram uma redução significativa na população patogênica composta por Peptostreptococcus, Comamonas e Fusobacterium , e uma expansão de Enterococcus e Proteobacteria na microbiota aderente à mucosa. Uma possível explicação é que os suplementos probióticos (B. longum, L. acidophilus e E. faecalis) alteram efetivamente a composição, a riqueza e a diversidade da microflora intestinal ao inibir certos patógenos em potencial, incluindo Fusobacterium e Peptostreptococcus, e aumentam o número de microorganismos benéficos.

Yazici C, et al. (2017) sugeriram que bactérias sulfidogênicas são um potencial fator de risco ambiental que contribui para o surgimento de CCR em afro-americanos (AA), já que estes têm maior risco em relação a brancos não-hispânicos $(\mathrm{BNH})$. Para isso, teorizaram que o sulfeto de hidrogênio, produzido por bactérias sulfidogênicas, que utilizam enxofre em seu metabolismo, pode ser um determinante, visto que contribui para oncogênese por desencadear vias pró-inflamatórias e ser genotóxico. Assim, compararam a abundância dessas bactérias na mucosa não acometida de pacientes com CCR em relação aos controles. Fusobacterium foi a bactéria sulfidogênica mais abundante identificada (XIE YH, et al., 2017). 
Xie $\mathrm{YH}$, et al. (2017) apresentaram o acréscimo gradual significativo de C. symbiosum no adenocarcinoma colorretal (ACR), CCR inicial e CCR avançado, tornando-o um marcador importante para triagem precoce de CCR, ampliando os horizontes do desenvolvimento de novos estudos para identificação de biomarcadores de neoplasia de cólon, principalmente em sua fase inicial.

O C. symbiosum apresentou uma predominância fecal acima dos marcadores teste imunoquímico fecal (TIF) e antígeno carcinoembrionário (ACE) convencional, como também, superior ao F. nucleatum, exibindo uma precisão maior na detecção de CCR precoce, permanecendo elevada em todos os tipos de tumores intestinais, atingindo $37-58 \%$ no adenoma avançado e 50\%-56\% no estágio inicial, assim, obtendo resultados duas vezes mais altos em comparação ao teste TIF e o ACE, como o aumento de $C$. symbiosum mostrou diferença significativa entre coorte de controle saudável e CCR precoce, e C. symbiosum alcançou área abaixo da curva mais alta para prever CCR precoce $(P<0,05)$ e adenoma avançado $(P<0,05)$, resultado que corrobora sua superioridade como um melhor marcador para discriminar a CCR é um marcador mais efetivo que o F. nucleatum na detecção de tumores colorretais.

É importante ressaltar a identificação de poder preditivo ao combinar C. symbiosum com TIF e ACE, aumentando a sensibilidade em 12 a $15 \%$, sem perda de especificidade, reconhecendo quase um terço dos pacientes com CCR em estágio inicial. Ao associar F. nucleatum e $C$. symbiosum combinados com TIF e ACE foi possível prever todos os pacientes com CCR com uma especificidade aprimorada de até $82 \%$ a $86 \%$ com especificidade de $69 \%$ a $87 \%$.

Dessa forma o estudo apresenta o $C$. symbiosum fecal é um biomarcador relevante e promissor para a identificação precoce e não invasiva de neoplasia colorretal, sendo mais eficaz do que marcadores relatados como $F$. nucleatum, TIF e ACE, como também pode ser utilizado de forma combinada, aumentando sua eficácia no diagnóstico não invasivo da CCR, além de apresentar uma precisão preditiva com custo aceitável, facilitando seu uso clínico em triagem precoce do CCR (XIE YH, et al., 2017).

Com base na análise dos artigos utilizados conforme mostra o Quadro 3 e os critérios de vieses aplicados, foi possível separá-los de acordo com a qualidade metodológica. Oito artigos foram analisados e apresentaram baixo risco de viés de seleção, performance, detecção, atrito, relato e outros, possuindo assim, boa qualidade metodológica. No entanto, outros 4 estudos apresentaram disparidades nos tipos de vieses, possuindo risco incerto por não relatarem alocação, randomização ou mascaramento. Ou alto risco de viés, sendo classificados como artigos que possuem baixa qualidade metodológica. 
Quadro 2 - Resumo dos resultados expostos nos artigos

\begin{tabular}{|c|c|c|c|}
\hline Autores & $\begin{array}{l}\text { Ano de } \\
\text { publicação }\end{array}$ & $\mathbf{N}$ total & Resultados \\
\hline Xiaoliang $\mathrm{X}$, et al. & 2019 & 140 & $\begin{array}{l}\text {-A suplementação com prebióticos em pacientes com } C C R \text { aumentou significativamente os níveis de transferrina ( } P \\
=0,027) \text {, IgG }(P=0,02) \text { e IgM }(P=0,00) \text { no pré-operatório (todos } P<0,05) \text {. } \\
\text {-No pós-operatório, foram identificados níveis aumentados de } \lg G(P=0,003) \text {, IgA }(P= \\
0,007) \text {, células } T \text { supressoras/citotóxicas }(C D 3+, C D 8+; P=0,043) \text { e linfócitos } B \text { totais }(C D 19+; P=0,012) \text { no grupo } \\
\text { prebiótico (todos } P<0,05) \text {. As comunidades bacterianas dominantes incluíam Bacteroidetes, Firmicutes, } \\
\text { Proteobacteria e Actinomycetes em diferentes grupos. }\end{array}$ \\
\hline Zaharuddin L, et al. & 2019 & 52 & $\begin{array}{l}\text {-52 Pacientes que fizeram uso de probióticos } 2 \text { vezes por dia durante } 6 \text { meses, iniciados } 4 \text { semanas após a cirurgia } \\
((\mathrm{P}<0,05) \\
\text {-Grupo placebo ( } \mathrm{n}=25) \text { e grupo controle com uso de probióticos Lactobacillus acidophilus, Lactobacillus lactis, } \\
\text { Lactobacillus casei subsp, Bifidobacterium longum, Bifidobacterium bifidum e Bifidobacterium infantil ( } \mathrm{n}=27 \text { ) } \\
\text { Apresentaram redução significativa no nível de citocinas pró-inflamatórias (TNF-a, IL-6, IL-10, IL-12, IL-17A, IL-17C } \\
\text { e IL-22), porém sem alteração considerável nos níveis citocinas anti-inflamatórias IFN-y em relação ao grupo controle. }\end{array}$ \\
\hline Watson $\mathrm{H}$, et al. & 2018 & 22 & $\begin{array}{l}\text {-A administração de } 4 \mathrm{~g} \text { por dia de ômega-3 (ácido eicosapentaenoico (EPA) e ácido docosahexaenoico (DHA) por } 8 \\
\text { semanas apresentou correlação com a proliferação de bactérias colônicas produtoras de butirato, substância com } \\
\text { efeitos antineoplásicos e anti-inflamatórios. }\end{array}$ \\
\hline Gao Z, et al. & 2015 & 22 & $\begin{array}{l}\text {-A administração de probiótico foi capaz de reduzir efetivamente as populações patogênicas de Fusobacterium e } \\
\text { Peptostreptococcus em pacientes com câncer colorretal. }\end{array}$ \\
\hline Pearson $\mathrm{T}$, et al. & 2019 & 401 & $\begin{array}{l}\text {-A relação entre a microbiota intestinal e a composição fecal dos ácidos biliares seria um fator de proteção para o } \\
\text { câncer colorretal. } \\
-1285 \text { pacientes que apresentavam pelo menos um adenoma colorretal, subdivididos em grupo UDCA (n=661) e } \\
\text { grupo placebo ( } n=624) \text {. } \\
\text {-Pacientes em uso de ácido ursodesoxicólico (UDCA) oral em } 8-10 \mathrm{mg} / \mathrm{kg} \text { por dia durante três anos apresentam } \\
\text { alterações significativas na microbiota composicional, sendo as dominantes Bacterioidetes e Firmicutes, não sendo } \\
\text { observadas no grupo placebo. } \\
\text {-UDCA pode reduzir o risco de adenoma colorretal, mas ainda precisa de mais investigação para determinar o } \\
\text { mecanismo associado a microbiota }\end{array}$ \\
\hline
\end{tabular}

REAS / EJCH | Vol.12(11) | e4672 | DOI: https://doi.org/10.25248/reas.e4672.2020 Página 7 de 11 


\begin{tabular}{|c|c|c|c|}
\hline Autores & $\begin{array}{l}\text { Ano de } \\
\text { publicação }\end{array}$ & $\mathbf{N}$ total & Resultados \\
\hline $\begin{array}{l}\text { PolakowskiCB, et } \\
\text { al. }\end{array}$ & 2018 & 73 & $\begin{array}{l}\text {-Descreveu o efeito da administração simbiótica em pacientes com câncer colorretal e concluiu que o seu uso por } 7 \\
\text { dias no pré-operatório atenua o estado inflamatório e está associado a reduções de morbidade, tempo de internação } \\
\text { hospitalar e uso de antibióticos nos pacientes portadores de CCR. }\end{array}$ \\
\hline Xie YH, et al. & 2017 & 1325 & $\begin{array}{l}\text {-O Clostridium symbiosum fecal é um biomarcador promissor para a detecção precoce e não invasiva de neoplasia } \\
\text { colorretal, sendo mais eficaz do que marcadores relatados como } F \text {. nucleatum, Teste Imunoquímico Fecal (FIT) e } \\
\text { Antígeno Carcinoembrionário sérico (CEA). - Foi encontrado aumento gradual significativo da abundância de } C \text {. } \\
\text { symbiosum no adenoma colorretal, CCR precoce e avançado }(P<0,01) \text { em comparação aos controles. } \\
\text {-A abundância relativa de } F \text {. nucleatum exibiu leve aumento no CCR precoce }(2,46 \text { vezes, } P=0,006) \text {, mas aumento } \\
\text { substancial no CCR avançado }(10,70 \text { vezes, } P<0,001) \text { em comparação com controles saudáveis. }\end{array}$ \\
\hline Bajramagic et al. & 2019 & 78 & $\begin{array}{l}\text {-O grupo tratado que usou probióticos de acordo com o esquema } 2 \times 1 \text { cápsulas a partir do terceiro dia de pós- } \\
\text { operatório durante os próximos trinta dias e, em seguida, } 1 \times 1 \text { por duas semanas a cada mês no próximo mês, } \\
\text { totalizando um ano, apresentou diminuição na morbidade pós-operatória tais como íleo e SII. }\end{array}$ \\
\hline Yazici C, et al. & 2017 & 329 & -Bactérias sulfidogênicas são um potencial fator de risco para o desenvolvimento de CCR eaicanos. \\
\hline Borresen S, et al. & 2016 & 29 & $\begin{array}{l}\text {-O grupo que consumiu farelo de arroz aumentou a riqueza bacteriana intestinal e revelou aumentos em três espécies } \\
\text { não identificadas anteriormente. Alterações no plasma, na urina e no metabolismo das fezes revelaram aumentos em } \\
\text { vários metabólitos microbianos, hospedeiros e derivados da dieta, como fitoesteróis, ácidos graxos, aminoácidos, } \\
\text { ácidos biliares e subprodutos de pequenas moléculas do metabolismo de carboidratos. Além disso, os extratos de } \\
\text { metabólitos das fezes humanas foram avaliados antes e após o consumo de farelo arroz ou feijão marinho para } \\
\text { observar um crescimento reduzido das células CRC. }\end{array}$ \\
\hline
\end{tabular}

Fonte: De Paula LMM, et al., 2020.

$$
\text { REAS / EJCH | Vol.12(11) | e4672 | DOI: https://doi.org/10.25248/reas.e4672.2020 Página } 8 \text { de } 11
$$


Revista Eletrônica Acervo Saúde / Electronic Journal Collection Health | ISSN 2178-2091

Quadro 3 - Qualidade metodológica dos estudos

\begin{tabular}{|c|c|c|c|c|c|c|}
\hline Autores & Viés de seleção & Viés de performance & Viés de detecção & Viés de atrito & Viés de relato & Outros vieses \\
\hline Xiaoliang X, et al., 2019 & Baixo risco & Baixo risco & Baixo risco & Baixo risco & Baixo risco & Baixo risco \\
\hline Zaharuddin L, et al., 2019 & Baixo risco & Baixo risco & Baixo risco & Baixo risco & Baixo risco & Baixo risco \\
\hline Watson $\mathrm{H}$, et al., 2018 & Baixo risco & Baixo risco & Alto risco & Risco incerto & Risco incerto & Risco incerto \\
\hline Gao Z, et al., 2015 & Risco incerto & Risco incerto & Risco incerto & Baixo risco & Baixo risco & Baixo risco \\
\hline Pearson T, et al., 2019 & Baixo risco & Risco incerto & Risco incerto & Baixo risco & Baixo risco & Baixo risco \\
\hline Polakowski CB, et al., 2018 & Baixo risco & Baixo risco & Baixo risco & Baixo risco & Risco incerto & Baixo risco \\
\hline Xie YH, et al., 2017 & Risco incerto & Risco incerto & Risco incerto & Risco incerto & Risco incerto & Risco incerto \\
\hline Bajramagic S, et al., 2019 & Risco incerto & Risco incerto & Baixo risco & Baixo risco & Baixo risco & Baixo risco \\
\hline Yazici C, et al., 2017 & Risco incerto & Risco incerto & Risco incerto & Baixo risco & Baixo risco & Baixo risco \\
\hline Borresen EC, et al., 2016 & Risco incerto & Risco incerto & Risco incerto & Risco incerto & Risco incerto & Risco incerto \\
\hline
\end{tabular}

Fonte: De Paula LMM, et al., 2020.

REAS / EJCH | Vol.12(11) | e4672 | DOI: https://doi.org/10.25248/reas.e4672.2020 Página 9 de 11 


\section{DISCUSSÃO}

Com base na leitura integral dos treze artigos para a presente revisão sistemática, foi possível observar de forma unânime que as Bifidobacterias spp e os Lactobacillus spp atuam reduzindo o risco de câncer de cólon e melhorando o tratamento e a recuperação no pós-operatória (BAJRAMAGIC S, et al., 2019). As Bifidobacterias spp têm seu benefício por restringir de maneira significativa o crescimento da neoplasia em três linhagens celulares distintas: Caco-2, SW 480 e HT-29 (ZAHARUDDIN L, et al., 2019).

Em relação aos Lactobacillus spp, identificou-se que a associação do Lactobacillus fermentum NCIMB 5221 com o Lactobacillus acidophilus ATCC 314 diminui a proliferação de células neoplásicas e do marcador de proliferação de células desreguladas do Câncer Colorretal (ZAHARUDDIN L, et al.,2019).

A microbiota intestinal é fundamental na regulação do desenvolvimento da resposta imune, sendo que a presença de bactérias não patogênicas inibe a proliferação das patogênicas (POLAKOWSKI CB, et al., 2018). Ademais, os probióticos possuem outros efeitos benéficos, como a adesão competitiva às membranas do epitélio e da mucosa intestinal, o revigoramento da barreira intestinal, além da alteração favorável da microbiota intestinal e a modulação do sistema imunológico (BAJRAMAGIC S, et al., 2019).

Ainda não se sabe ao certo quais são as espécies bacterianas que mais influenciam na evolução do câncer colorretal. No entanto, de acordo com Tilg H, et al. (2018); existem evidências que comprovam uma importante participação de Escherichia coli, Fusobacterium nucleatum e Bacteroides fragilis. Segundo Xie $\mathrm{YH}$, et at. (2017); as determinações dos perfis de microbiota feitos através de sequenciamentos laboratoriais podem ser eficazes na previsão e prevenção de câncer colorretal (CCR).

Nesse sentido, a linhagem das Fusobacterium nucleatum tem se mostrado um potencial marcador para detecção desta neoplasia uma vez que Tilg $\mathrm{H}$, et al. (2018); evidenciou aumento da presença desta bactéria na displasia de alto grau e no CCR estabelecido. Além disso, a grande quantidade de Fusobacterium nucleatum no tecido cancerígeno também refletiu em uma redução da sobrevida nos pacientes portadores da neoplasia, podendo atuar como potencial marcador prognóstico da doença.

Apesar de o Fusobacterium nucleatum ser uma das cepas bacterianas mais prevalentes nos tecidos com câncer colorretal (CCR) e sua importância ser sustentada por estudos clínicos e pré-clínicos, as evidências para Escherichia coli são baseadas principalmente em investigações pré-clínicas. Naturalmente, as Escherichia coli são comensais intestinais, no entanto certas cepas adquirem a capacidade de promover inflamação intestinal e produzir toxinas como a colibactina, que possui um potencial oncogênico. Além disso, foi relatado que a Escherichia coli associada à mucosa é significativamente mais prevalente no tecido CCR e correlaciona-se com o estágio e o prognóstico do tumor (TILG H, et al., 2018).

Semelhantemente à Escherichia coli, evidências experimentais relatam o papel de Bacteroides fragilis na neoplasia intestinal. A microbiota comensal é composta em cerca de $1 \%$ a $2 \%$ por essa bactéria, sendo sua toxina responsável por causar diarreia inflamatória e tumorigênese relacionada à inflamação (TILG H, et al., 2018). Sendo assim, na maioria dos estudos avaliados, foi sugerido que a microbiota intestinal também possui um potencial efeito carcinogênico na região colorretal. Essa microbiota específica seria a marcada pela baixa diversidade, presença de gêneros produtores de ácido graxo de cadeia curta e de patobiontes putativo, exemplificados pelo Eubacterium, Roseburia e Fusobacterium nucleatum (WATSON H, et al., 2017).

Segundo Yazici C, et al. (2017), bactérias específicas, como a Bilophila Wadsworthia , a Fusobacterium nucleatum e as bactérias redutoras (Desulfovibrio spp), produzem o gás sulfídrico (H2S), responsável pela indução de vias proliferativas e pró-inflamatórias que levam à restrição da oxidação do butirato, substrato das células pertencentes ao cólon e metabólito anticarcinogênico. Além disso, esse estudo evidenciou que a presença dessas bactérias sulfidogênicas têm correlação epidemiológica com o câncer colorretal, uma vez que estão presentes em quantidade dez vezes maior na mucosa colônica da população afrodescendente comparada à população branca não hispânica nos Estados Unidos (OLIVEIRA M, et al., 2018).

Todos os artigos revisados compreenderam ensaios clínicos sem perda de seguimento ou interrupção precoce do estudo por algum benefício, sendo que em apenas dois não houve randomização e cegamento dos participantes e avaliadores. No que tange aos vieses, apenas um artigo apresentou alto risco de viés de detecção. 
Desses outros, quatro apresentaram risco incerto e sete apresentaram baixo risco para viés de seleção, de detecção, de relato e de atrito, enquanto cinco apresentaram risco incerto e seis baixo risco para viés de performance. Com isso, é possível concluir que as evidências obtidas das análises possuem um bom suporte metodológico e foi possível identificar concordância em vários aspectos já citados, não tendo nenhum deles apresentado desfechos discrepantes em relação ao outro.

\section{CONSIDERAÇÕES FINAIS}

As bactérias Escherichia coli, Fusobacterium nucleatum e Bacteroides fragilis estão consistentemente associadas ao câncer colorretal e têm potencial no diagnóstico por atuarem como marcadores da neoplasia. Além disso, a microbiota intestinal exemplificada por Eubacterium, Roseburia e Fusobacterium nucleatum, possui um potencial efeito carcinogênico na região colorretal. No entanto, os estudos também apresentaram colônias bacterianas capazes de reduzir o risco de câncer de cólon e melhorar o tratamento como as Bifidobacterias spp e os Lactobacillus, que atuam na recuperação no pós-operatório. Ademais, o Clostridium symbiosum, um biomarcador promissor para a detecção precoce e não invasiva, demonstra ser possível iniciar o tratamento em estágios iniciais e diminuir a morbimortalidade da patologia. Esse estudo mostra que os suplementos prebióticos, probióticos e ácidos graxos alteram a microbiota intestinal, sendo propícios para prevenção e tratamento do câncer colorretal.

\section{REFERÊNCIAS}

1. BAJRAMAGIC S, et al. Usage of Probiotics and its Clinical Significance at Surgically Treated Patients Sufferig from Colorectal Carcinoma. MED ARCH, 2019; 73(5):316-320.

2. BORRESEN EC, et al. A randomized-controlled trial to increase navy bean or rice bran consumption in colorectal cancer survivors. Nutr Cancer, 2016; 68:1269-1280.

3. BRASIL. Ministério da Saúde. Secretaria de Ciência, Tecnologia e Insumos Estratégicos. Departamento de Ciência e Tecnologia. Diretrizes metodológicas : elaboração de revisão sistemática e metanálise de ensaios clínicos randomizados, Brasilia, 2012.

4. CAMPOS FGCM, et al. Incidência de câncer colorretal em pacientes jovens. Rev. Col. Bras. Cir., 2017; 44(2): $208-215$.

5. DE CARVALHO A, et al. Avaliação do risco de viés de ensaios clínicos randomizados pela ferramenta da colaboração Cochrane. Diagn Tratamento, 2013; 18(1): 38-44.

6. ESTATÍSTICA DO CÂNCER. 2020. In Instituto nacional do câncer. Brasil: Ministério da saúde. Disponível em: https://www.inca.gov.br/numeros-de-cancer. Acesso em: 26 abril 2020.

7. CANCER. 2018. In: Organização Pan-Americana de Saúde (OPAS/OMS). Brasília: OPAS. Disponível em: https://www.paho.org/bra/index.php?option=com_content\&view=article\&id=5588:folha-informativa-cancer\&lt emid=1094. Acesso em: 26 abril 2020.

8. GALVÃO TF, et al. Principais itens para relatar Revisões sistemáticas e Meta-análises: A recomendação PRISMA. Epidemiologia e Serviços de Saúde, 2015; 24: 335-342.

9. GAO Z, et al. Probiotics modify human intestinal mucosa-associated microbiota in patients with colorectal cancer. Molecular medicine reports, 2015; 12: 6119-6127.

10. OLIVEIRA M, et al. Disparidades na mortalidade de câncer colorretal nos estados brasileiros. Revista Brasileira de Epidemiologia, 2018; 21: 1800-1812.

11. PARK Y, et al. Ingestão e mortalidade de fibras alimentares no estudo de dieta e saúde NIH-AARP. Arch Intern Med. 2011; 171(12): 1061-1068.

12. PEARSON T, et al. Effects of ursodeoxycholic acid on the gut microbiome and colorectal adenoma development. Cancer Med, 2019; 8: 617-628.

13. PELIZZER T, et al. Prevalência de câncer colorretal associado ao papilomavírus humano: uma revisão sistemática com metanálise. Rev. bras. epidemiol., 2016; 19 (4): 791-802.

14. POLAKOWSKI CB, et al. Impact of the preoperative use of synbiotics in colorectal cancer patients: a prospective, randomized, double-blind, placebo-controlled study. The End-to-end Journal, 2018; 58: 40-46.

15. REZASOLTANI S, et al. The association between fecal microbiota and different types of colorectal polyp as precursors of colorectal câncer. Microbial Pathogenesis, 2018; 124: 244-249.

16. SCHWABE RF, JOBIN C. The microbiome and cancer. Nature Reviews Cancer, 2013; 13(11): 800-812.

17. SILVA MD, ERRANTE PR. Câncer colorretal: fatores de risco, diagnóstico e tratamento. Revista UNILUS Ensino e Pesquisa, 2016; 33(13): 133-140.

18. TILG H, et al. The intestinal microbiota in colorectal cancer. Cancer cell, 2018; 33(6): 954-964

19. WATSON H, et al. A randomised trial of the effect of omega-3 polyunsaturated fatty acid supplements on the human intestinal microbiota. Gut, 2018; 67: 1974-1983.

20. WAGNER NRF, et al. Mudanças na microbiota intestinal e uso de probióticos no pós-operatório de by-pass gástrico em Yde-Roux e gastrectomia vertical Sleeve: uma revisão integrativa. ABCD Arq Bras Cir Dig, 2018; 31(4):e1400.

21. XIAOLIANG $X$, et al. Effects of prebiotics on immunologic indicators and intestinal microbiota structure in perioperative colorectal cancer patients. Nutrition, 2019; 61: 132-142.

22. XIE YH, et al. Fecal Clostridium symbiosum for Noninvasive Detection of Early and Advanced Colorectal Cancer: Test and Validation Studies. EBioMedicine, 2017; 25: 32-40.

23. YAZICI C, et al. Race-dependent association of sulfidogenic bacteria with colorectal cancer. Gut, 2017; 66: $1983-1994$.

24. ZAHARUDDIN L, et al. A randomized double-blind placebo-controlled trial of probiotics in post-surgical colorectal cancer. BMC Gastroenterology, 2019; 19: 131. 